\title{
Stem cells: a new way of therapy for cardiovascular disorders
}

\author{
Rabia Amjad, Isha Ishtiaq, Noor Fatima \\ Department of Biotechnology, University of Sialkot, Sialkot, Pakistan \\ Contributions: (I) Conception and design: None; (II) Administrative support: None; (III) Provision of study materials or patients: None; (IV) \\ Collection and assembly of data: None; (V) Data analysis and interpretation: None; (VI) Manuscript writing: All authors; (VII) Final approval of \\ manuscript: All authors. \\ Correspondence to: Rabia Amjad. Department of Biotechnology, University of Sialkot, Sialkot, Pakistan. Email: Rabiaamjad932@gmail.com.
}

\begin{abstract}
Cardiovascular disorder affects the overall health of an individual and hence the quality of life. Stem cell therapy involves the use of stem cells widely used to treat different conditions. People having severe cardiovascular disorder can be treated with stem cells by generating heart muscles, stimulating the growth of blood vessels and by the secretion of different growth factors. Different types of stem cells are used for cardiac repair. Adipose stem cells and induced pluripotent stem cells are better options for increasing the survival rate. In this review we will discuss different types of stem cells, their activation pathway, generation, hurdles in transplantation and how to overcome them and their applications.
\end{abstract}

Keywords: Cardiovascular disorders; induced pluripotent stem cells (IPSC's); cardiac dysfunction; ASC's; viability

Received: 25 December 2019; Accepted: 16 October 2020; Published: 03 November 2020.

doi: $10.21037 /$ sci-2019-048

View this article at: http://dx.doi.org/10.21037/sci-2019-048

\section{Introduction}

Cardiovascular diseases are those severe and life threatening disorders that reduce the life span of a person and ultimately affect the quality of its life (1). In spite of the establishment in medicine and surgery, it remains the biggest threat that lead to increase in mortality rate (2). Globally the estimation of population that is being affected is about 40 million (1). Ventricular filling or blood ejection are followed by restlessness, fatigue, congestion and edema are some major causes of heart failure $(1,3)$. The heart muscle is totally destroyed by Ischemia, swelling and remodelling after the heart attack (4). One of the wonders of the modern era is Stem cell therapy which becomes the solution of our problem involves characterization, isolation, expansion and differentiation of these cells and give us a proper way to treat this epidemic (4). Stem cells and the exosomes that are derived from it helps in repairing the damaged tissue of heart (5). Embryonic and adult stem cells are effective in this regard, where they further differentiate into induced pluripotent stem cells (IPSC's), adipose tissue stem cells, Cardiac stem cells and Umbilical cord stem cells (1). The effective myocardial stem cell therapy is responsible for the replacement of damaged tissue to the functionalized ones. Organ formation, development of embryogenesis and tissue regeneration are the vital functions of stem cells. They are capable of 'self-renewing' and have the ability of forming vessels and tissues (6). The three germ layers are formed by the stem cells (7). In the recent studies it has been found that the stem cells of adipose tissue have the potential of restoring cardiac function (2). Although there are some limitations like relative immaturity, graft retention in sufficient cellular proliferation and small graft size, yet it is a novel option for treating this disorder (8).

\section{Classification of stem cells}

Stem cells are categorized on the basis of their potential and site from where they are obtained or harvested.

(I) Pluripotent stem cells are those cells that divide and regenerate into three germ layers and in all types of cells of the adult except extra embryonic tissues

(II) Totipotent stem cells have the potential to form three germ layers and along with extra embryonic 
tissues.

(III) Multipotent stem cells differentiate into a narrow and restricted range of cells.

(IV) Unipotent stem cells are those cells that established into one type of cells (9).

Heart failure leads the life of a healthy person to a drastic level. Recent study in different types of cardiomyopathies helps us and gives approach to treat this disorder by simultaneous revascularization and stem cell therapy. Stem cells work efficiently when they are responsive to their comorbidities (1).

\section{Stem cells used in cardiac regeneration therapy}

The different types of stem cells now differentiate into cardiac cells as shown below in (Figure 1).

\section{Skeletal muscle cells}

These cells are taken from the basil lamella of skeletal muscle fibre. They improved the heart functioning because they easily form myotubes and does not involved in ventricular remodelling. Recent investigation showed that due to the absence of a junction gap they failed to generate arrhythmias, thus not a suitable option for cardiomyopathy (1).

\section{Induced pluripotent stem cells}

We used transcriptional factor to for obtain these cells from adult fibroblast. They are having the same characteristics and structure as that of embryonic stem cells so it gives a benefit in this case. They restore the cardiac function by integrating into it. The nonspecific pluripotent should be removed to maintain homogeneity and specificity (1).

\section{Adipose derived mesenchymal stem cells}

An attractive approach for obtaining stem cells by easy harvesting procedure. Study on rodents gives us beneficial results but not evident in humans (1). The cardiac repair through these adipose derived stem cells is effective because of its paracrine effect. Rather than differentiation into lineage cardiac cells; anti apoptosis, pro-angiogenesis and inhibition of fibrosis make them a better option, but they have problem of low cardiac retention and survival rate which are affected because of rejection after transplantation. Researchers have proposed a solution to this serious problem by confirming the secretion of paracrine through various strategies involving genetic, pharmacological, physiological, physical and cytokine pre conditioning and tissue engineering to allow ACS's to release the secretome $(2,10)$.

\section{Cardiac stem cells}

Investigation on animal models shows that they improved the left ventricular function by expressing c-kit. They form clusters of cardiospheres by the suspension culture in vitro. A good yield had been obtained during animal and human investigations $(1,11)$.

\section{Embryonic stem cells}

They show similarity to pluripotent cells but they are actually inner mass cells of the human embryo which are undifferentiated. They can easily establish ectoderm, endoderm and mesoderm. Cardiac cells are easily derived from them but due to some ethical issue their use is restricted. The immune system is also responsive to it. Moreover, genetic stability is also a major issue that we face (1). In a study in rat hearts, we come to know that these cells result in increase in contractility, improvement in the structure of myofibril and proper handling of calcium in engineered heart tissues. They are less stiff as compared to MSC's so enhance the graft size and improve the function (8).

\section{Mesenchymal stem cells}

Mesenchymal stem cells therapy has saved many lives. In an experiment in which we use three data base and metaanalysis is performed on outcomes of death, readmission, the 6-min walk test (6MWT), New York Heart Association (NYHA) class and left ventricular ejection function (LVEF). Six hundred and twelve patients are treated with MCS's having death rate reduction $36 \%$. In readmission there is a reduction of $34 \%$. An improvement of $40.44 \mathrm{~m}$ in $6 \mathrm{MWT}$ and in NYHA and LVEF groups $95 \%$ and $5.25 \%$ reduction is observed (3). Moreover, MSC's treated with plateletrich plasma has a greater influence than MSC's alone (12). Vesicles derived from MSC's are used for the treatment of many disorders (13).

\section{Cortical bone stem cells}

We have evident in an experiment on mini-swine that if 


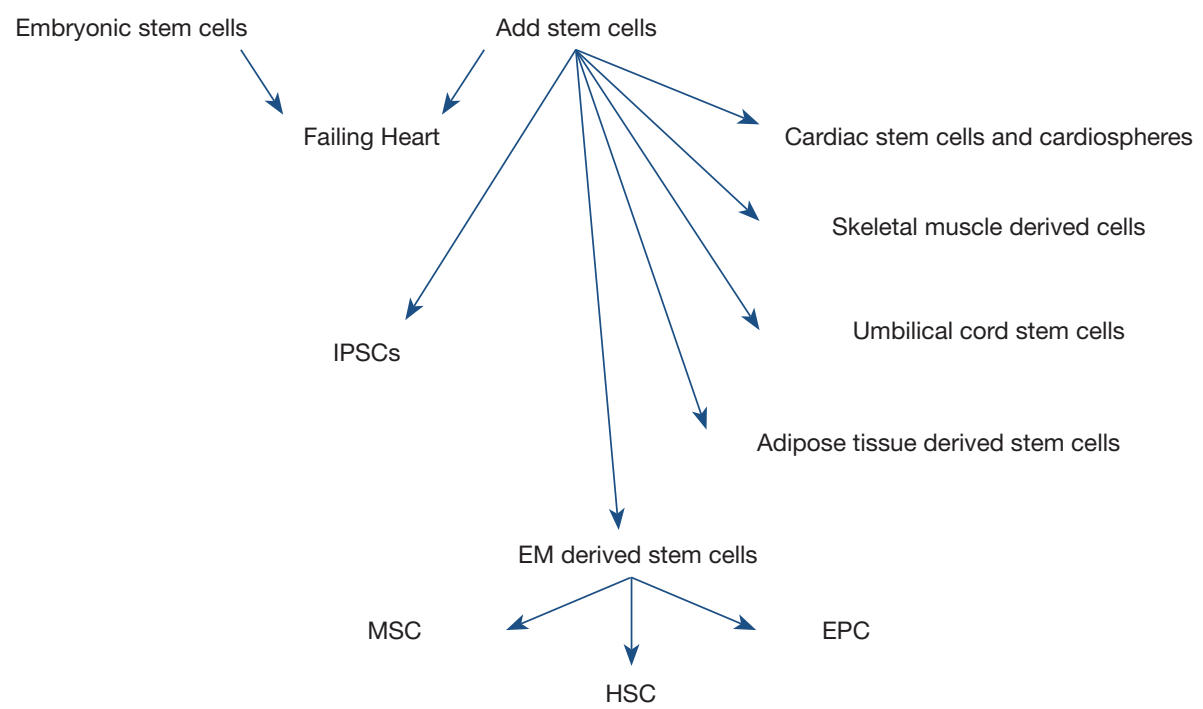

Figure 1 Types of stem cells for cardiac regeneration. Embryonic and adult stem cells are the two alternatives for a failing heart survival. Adult stem cell is further classified into cardiac stem cells, skeletal muscle stem cells, umbilical cord stem cells, adipose tissues and iPSC's. The CM derived stem cells are further divided into MSc, HSC and EPC's. iPSC's, induced pluripotent stem cells; CM, cardio myocytes; EPC's, endothelial progenitor cells.

we use cortical bone stem cells after myocardial infraction can increase the pump function and decrease the scar size. CBSC's leads to the death of cardio myocytes (CM) and non-CM. It also involves in the formation of immune cells after myocardial injury (14).

\section{Activation and regulation of stem cells}

Stem cells play a significant role throughout the lives. Nearly all types of tissues of brain heart, skin and organs are formed through them. This mechanism is greatly affected due to the loss of stem cell activity. It is evident that stem cell activity is significantly depend on activation mechanism and cellular signalling that involved cytokines (i17, il23, G-CSF), metabolic factors, extracellular matrix and supporting cells that lead to improve the mobilization mechanism otherwise this irregular impairment can lead to heart failure $(15,16)$.

\section{Induced pluripotent stem cells and cardiovascular disease}

IPSC's increase the survival of tissue and neighbouring cells through paracrine effects. Investigations have shown that extracellular vesicles derived from these stem cells undergo intercellular signalling and communication and are affective in survival of CM and prevent them from apoptosis. These vesicles become a promising approach for drug delivery and immunotherapy (1). Hypertrophic cardiomyopathy is caused due to the mutation that code sarcomeric protein results in abnormal thickness of left ventricular wall and interventricular septum that lead to high risk of heart death. For studying such a serious disorder only animals' models are available. So in this case we used induced pluripotent stem cells to form cardiac tissues and studied the mutation of gene in them. It is observed that there is a mutation in R326Q in MYBPC3 gene which is the main reason of this cardiomyopathy and easily studied by IPS's (17).

\section{Approaches for cardiac regeneration}

In experimental studies on zebra fish, newt and neonatal mice it is observed that adult mammalian CM differentiated and proliferative in a limited range. When fibrous scar tissues are used for repairing damage heart they lead to contraction reduction and ultimately heart failure (18). So for repairing heart an approach of using CMs derived from PSC's become a promising alternative as shown in Figure 2. Human CMs have the capacity to rapidly proliferate less than one percent per year therefore adult CMs are also prove better in this regard (19). 


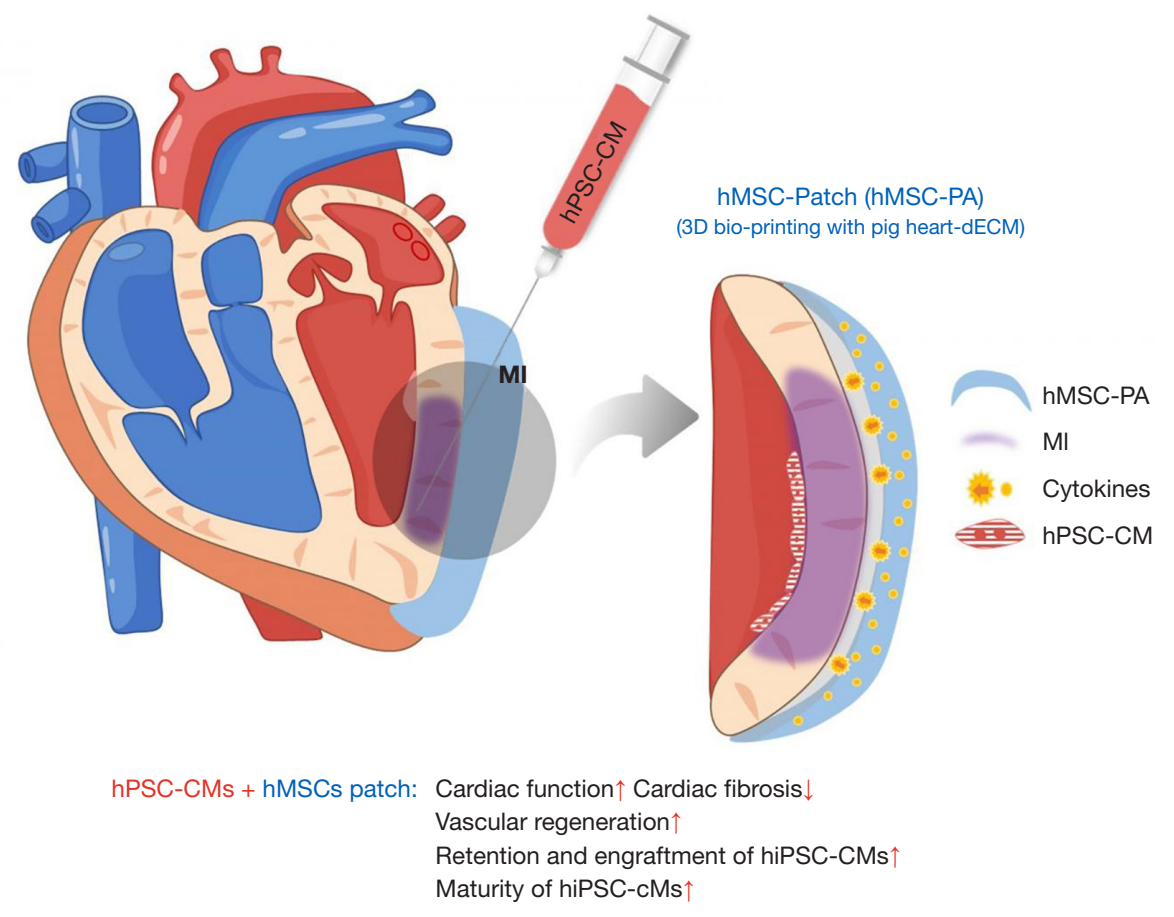

Figure 2 CM Derived PSC's an alternative approach for cardiac regeneration therapy. It demonstrated hMSC loaded patch implantation cause the maturation of hPSC-CMs. The cells become elongated, rectangular and organized developed into mature adult CM's. Functional maturation of intramyocardially injected hiPSC-CMs is particularly important. This reduces the potential risk of arrhythmias, which is a major cause of sudden cardiac death. CM, cardio myocytes.

\section{The generation of PSC-CMs}

Two methods are currently available for the generation of CM from PSCs. They are adherent and suspension cultures. Growth factors or small molecules such as Wnt modulators, bone morphogenetic protein 4 and activin A are all needed for proper cardiac tissue development. After 10 days of differentiation, the cells start working, but priorly elimination of undifferentiated cells is necessary. For purification surface markers and glucose depletion with lactate supplement are needed (19).

\section{Preclinical transplantation studies of PSC-CMs}

Dispersed and tissue engineered cells both methods are used for transplantation. The former can be easily prepared, cryopreserved and directly injected, but it has a serious disadvantage that it leaks from the site of administration and do not retain after transplantation. On the other hand, the tissue engineered cells do not lost and have the better chance of survival and retention (19).

\section{Hurdles for clinical applications}

The chances of arrhythmias after the transplantation of cardiac tissue, automaticity and rejection by the immune system are still the hurdles that we have to face during our clinical application (19).

\section{Improvement in cardiac dysfunction}

One of the types of cardiomyopathy is Arrhythmogenic right ventricular cardiomyopathy (ARVC) which is inherited and caused by fibrofatty replacement. It results in cardiac dysfunction. It is evident that type 5 is caused by p.S358L mutation in trans membrane protein 43 . In a study we observe the transgenic mice TMEM over the expression cause death. The replacement is caused due to TMEM mutation. For treatment, over the expression of $\mathrm{AB} 1$ and GSK3B inhibition can lead to prevention of cardiac function (20).

Another study showed that two groups Exo and LExo having exosome from bone marrow derived mesenchymal 
cells can lead to increase in M2 polarization and decrease in M1 polarization due to LPS. The M2 macrophages involve post-infraction inflammation and apoptosis of CM which draw a conclusion that a low concentration of exosome is an appropriate treatment after myocardial infraction $(21,22)$.

\section{Tracking of viability of stem cells}

Using imaging tools, we can detect and check the whether the stem cell is feasible or not, which greatly affect cardiac repair. Nanoparticles are used for photoacoustic imaging. A contrasting agent for this purpose is Nano rod of gold that are coated with IR775C, a reactive oxygen species (ROS) that show sensitivity in the infrared region. When cell degrades it produces ROS when we compare the signals of IR775C to ROS, we can easily tract the stem cell location. It involves longitudinal tracking (23).

\section{Cardioprotective affect for the stem cells}

When cells are engrafted, they are mostly destroyed by the oxidative environment, which ultimately reduces the effectiveness of the procedure. In a clinical trial it is observed that Pre-Incubation with omega-3 fatty acid including, eicosapentaenoic acid (EPA) and docosahexaenoic acid (DHA) and ascorbic acid (AA) before hydrogen peroxide treatment protects the embryonic stem cells along with improvement in fibrosis. It also increases cell viability before transplantation (24).

\section{Applications}

\section{Pharmacological studies}

IPSC's-ECs provide us an opportunity to study the effect of different drugs on them. Pravastatin response to them is observed and normal and obese mice. Anti-hypersensitive drugs are also investigated which showed resistance to hypertension in CM and vascular smooth muscles derived from IPSC's $(7,25)$.

\section{Organ on a chip}

It is a three dimensional cell culture chip that is used for research in tissue engineering, fabrication and formation of human organs using human cells. Due to lack of model stem cells have become a source in this regard. It is form various systems such as human vascular micro physiological system to study the human disease, drug responses and three dimensional vascular network engineering (7).

\section{Graft engineering}

Vascular and valvular grafts are produced by using stem cells that are durable and affective. Moreover they are non-immunogenic and are best suited for cardiovascular disorders. They are also involved in whole organ graft engineering $(7,25)$.

\section{Conclusions}

Stem cell therapy is widely used in many types of studies and research and in different types of cardiomyopathies. Different protocols and procedures are available and their introduction in living system and prevention from immune rejection is a challenging task. This therapy is also affected by a number of factors. Different types of stem cells are used for the generation of cardiac tissues and hence in this era of modern medicine stem cells become a wonder to treat and cure a number of patients. And in near future use of nanoparticles for its tracking make it a better option.

\section{Acknowledgments}

Funding: None.

\section{Footnote}

Conflicts of Interest: All authors have completed the ICMJE uniform disclosure form (available at http://dx.doi. org/10.21037/sci-2019-048). The authors have no conflicts of interest to declare.

Ethical Statement: The authors are accountable for all aspects of the work in ensuring that questions related to the accuracy or integrity of any part of the work are appropriately investigated and resolved.

Open Access Statement: This is an Open Access article distributed in accordance with the Creative Commons Attribution-NonCommercial-NoDerivs 4.0 International License (CC BY-NC-ND 4.0), which permits the noncommercial replication and distribution of the article with the strict proviso that no changes or edits are made and the original work is properly cited (including links to both the formal publication through the relevant DOI and the license). 
See: https://creativecommons.org/licenses/by-nc-nd/4.0/.

\section{References}

1. Nair N, Gongora E. Stem cell therapy in heart failure: Where do we stand today? Biochim Biophys Acta Mol Basis Dis 2020;1866:165489.

2. Li X, Ma T, Sun J, et al. Harnessing the secretome of adipose-derived stem cells in the treatment of ischemic heart diseases. Stem Cell Res Ther 2019;10:196.

3. Fan M, Huang $Y$, Chen $Z$, et al. Efficacy of mesenchymal stem cell therapy in systolic heart failure: a systematic review and meta-analysis. Stem Cell Res Ther 2019;10:150.

4. Klopsch C, Gaebel R, Lemcke H, et al. VimentinInduced Cardiac Mesenchymal Stem Cells Proliferate in the Acute Ischemic Myocardium. Cells Tissues Organs 2018;206:35-45.

5. Huang P, Wang L, Li Q, et al. Combinatorial treatment of acute myocardial infarction using stem cells and their derived exosomes resulted in improved heart performance. Stem Cell Res Ther 2019;10:300.

6. Mardanpour P, Nayernia K, Khodayari S, et al. Application of Stem Cell Technologies to Regenerate Injured Myocardium and Improve Cardiac Function. Cell Physiol Biochem 2019;53:101-20.

7. Jang S, Collin de l'Hortet A, Soto-Gutierrez A. Induced Pluripotent Stem Cell-Derived Endothelial Cells: Overview, Current Advances, Applications, and Future Directions. Am J Pathol 2019;189:502-12.

8. Bargehr J, Ong LP, Colzani M, et al. Epicardial cells derived from human embryonic stem cells augment cardiomyocyte-driven heart regeneration. Nat Biotechnol 2019;37:895-906.

9. Huynh K. Stem cell therapy improves heart function by triggering an acute immune response. Nat Rev Cardiol 2020;17:69.

10. Ibarra-Ibarra BR, Franco M, Paez A, et al. Improved efficiency of cardiomyocyte-like cell differentiation from rat adipose tissue-derived mesenchymal stem cells with a directed differentiation protocol. Stem Cells Int 2019;2019:8940365.

11. Witman N, Zhou C, Grote Beverborg N, et al. Cardiac progenitors and paracrine mediators in cardiogenesis and heart regeneration. Semin Cell Dev Biol 2020;100:29-51.

12. Zaki SM, Algaleel WA, Imam RA, et al. Mesenchymal stem cells pretreated with platelet-rich plasma modulate doxorubicin-induced cardiotoxicity. Hum Exp Toxicol 2019;38:857-74.

13. Mohammadi MR, Riazifar M, Pone EJ, et al. Isolation and characterization of microvesicles from mesenchymal stem cells. Methods 2020;177:50-7.

14. Hobby ARH, Sharp TE 3rd, Berretta RM, et al. Cortical bone-derived stem cell therapy reduces apoptosis after myocardial infarction. Am J Physiol Heart Circ Physiol 2019;317:H820-H829.

15. Gemery JM, Forauer AR, Hoffer EK. Activation of stem cell up-regulation/mobilization: a cardiovascular risk in both mice and humans with implications for liver disease, psoriasis and SLE. Vasc Health Risk Manag 2019;15:309-16.

16. Bick AG, Pirruccello JP, Griffin GK, et al. Genetic Interleukin 6 Signaling Deficiency Attenuates Cardiovascular Risk in Clonal Hematopoiesis. Circulation 2020;141:124-31.

17. Dementyeva EV, Medvedev SP, Kovalenko VR, et al. Applying Patient-Specific Induced Pluripotent Stem Cells to Create a Model of Hypertrophic Cardiomyopathy. Biochemistry (Mosc) 2019;84:291-8.

18. Li Z, Hu S, Cheng K. Chemical Engineering of Cell Therapy for Heart Diseases. Acc Chem Res 2019;52:1687-96.

19. Kadota S, Shiba Y. Pluripotent Stem Cell-Derived Cardiomyocyte Transplantation for Heart Disease Treatment. Curr Cardiol Rep 2019;21:73.

20. Padrón-Barthe L, Villalba-Orero M, GómezSalinero JM, et al. Severe Cardiac Dysfunction and Death Caused by Arrhythmogenic Right Ventricular Cardiomyopathy Type 5 Are Improved by Inhibition of Glycogen Synthase Kinase-3 $\beta$. Circulation 2019;140:1188-204.

21. Xu R, Zhang F, Chai R, et al. Exosomes derived from pro-inflammatory bone marrow-derived mesenchymal stem cells reduce inflammation and myocardial injury via mediating macrophage polarization. J Cell Mol Med 2019;23:7617-31.

22. Patil M, Henderson J, Luong H, et al. The Art of Intercellular Wireless Communications: Exosomes in Heart Disease and Therapy. Front Cell Dev Biol 2019;7:315.

23. Dhada KS, Hernandez DS, Suggs LJ. In Vivo Photoacoustic Tracking of Mesenchymal Stem Cell Viability. ACS Nano 2019;13:7791-9.

24. Shabani P, Ghazizadeh Z, Gorgani-Firuzjaee S, et al. Cardioprotective effects of omega- 3 fatty acids and 
ascorbic acid improve regenerative capacity of embryonic stem cell-derived cardiac lineage cells. Biofactors 2019;45:427-38.

doi: $10.21037 /$ sci-2019-048

Cite this article as: Amjad R, Ishtiaq I, Fatima N. Stem cells: a new way of therapy for cardiovascular disorders. Stem Cell Investig 2020;7:19.
25. Ge F, Wang Z, Xi JJ. Engineered maturation approaches of human pluripotent stem cell-derived ventricular cardiomyocytes. Cells 2019;9:9. 